\title{
Order-disorder transitions of Cu-Al-Mn shape-memory alloys
}

\author{
Eduard Obradó, Carlos Frontera, ${ }^{*}$ Lluís Mañosa, and Antoni Planes \\ Departament d'Estructura i Constituents de la Matèria, Facultat de Física, Universitat de Barcelona. Diagonal, 647, \\ E-08028 Barcelona, Catalonia, Spain
}

(Received 8 October 1997)

\begin{abstract}
The order-disorder transitions in $\mathrm{Cu}-\mathrm{Al}-\mathrm{Mn}$ shape-memory alloys have been studied experimentally by means of calorimetric techniques. Results are compared with Monte Carlo simulations of a simplified BlumeEmery-Griffiths model. A first order $\mathrm{DO}_{3} \rightleftharpoons A 2$ transition is obtained for the stoichiometric $\mathrm{Cu}_{3} \mathrm{Al}$ alloy, while for concentrations near $\mathrm{Cu}_{2} \mathrm{AlMn}$ two second order transitions are found: $L 2_{1} \rightleftharpoons B 2$ and $B 2 \rightleftharpoons A 2$. Despite the simplicity of the model, the agreement between experimental and simulation results is remarkably good. Finally, the metastable phase diagram of bcc Cu-Al-Mn alloys is presented. [S0163-1829(98)05045-0]
\end{abstract}

\section{INTRODUCTION}

Cu-based alloys are, as most noble-metal-based alloys, electron or Hume-Rothery compounds. For these systems, the stability of the different phases at different temperatures and concentrations depends, essentially, on the average number of conduction electrons per atom $(e / a) .{ }^{1}$ For an $e / a$ ratio near 1.5 , these alloys crystallize at high temperatures into the bcc structure (or $\beta$ phase). For kinetic reasons, this $\beta$ phase can be retained below its stability limit by means of a suitable (fast enough) cooling. The metastable $\beta$ phase orders during cooling and, for certain compositions (which are those of interest in this work), undergoes at a lower temperature a first-order, diffusionless, structural transition to a more close-packed phase. This transition is known as the martensitic transformation, ${ }^{2}$ and has received a great deal of attention in recent years because it is the origin of shape memory properties, which confer a considerable technological importance on these systems. ${ }^{3}$

This paper is concerned with the study of the metastable phase diagram of the $\mathrm{Cu}-\mathrm{Al}-\mathrm{Mn}$ alloy system. Compared with other $\mathrm{Cu}$-based ternary alloys, this system has some interesting properties due to the presence of manganese.

(1) Mn makes the $\beta$ (bcc) phase more stable to diffusional decomposition than it is in other $\mathrm{Cu}$-based alloys and slows down this decomposition when the $\beta$ phase becomes metastable. ${ }^{4,5}$ Because of this, more adequate shape-memory properties can be obtained, and $\mathrm{Cu}$ $\mathrm{Al}-\mathrm{Mn}$ or $\mathrm{Cu}-\mathrm{Al}-\mathrm{Mn}$-based alloys have been preferred for some technological applications rather than other $\mathrm{Cu}-$ based alloys.

(2) $\mathrm{Cu}-\mathrm{Al}-\mathrm{Mn}$ alloys have magnetic ordering properties, whose specific features depend on the crystallographic phase and the composition of each alloy. Thus, the Heusler alloy $\mathrm{Cu}_{2} \mathrm{MnAl}$ is ferromagnetic, with a relatively high Curie temperature $(630 \mathrm{~K}),{ }^{5,6}$ and $\mathrm{Cu}_{3} \mathrm{Mn}_{2} \mathrm{Al}$ is antiferromagnetic. ${ }^{7}$ These properties are due to the RKKY interaction ${ }^{8}$ among the Mn atoms of the crystalline lattice. The magnetic degrees of freedom make $\mathrm{Cu}$ Al-Mn alloys very interesting from a fundamental point of view, since the interaction between magnetic, elastic and configurational properties can be studied for these alloys.

From the equilibrium phase diagram of these alloys ${ }^{9}$ (Fig. $1)$, it can be seen that, for compositions close to the $\mathrm{Cu}_{3-x} \mathrm{AlMn}_{x}$ line, the disordered $\beta$ phase ( $A 2$ or bcc structure, space group $\operatorname{Im} 3 \mathrm{~m}$ ) is the equilibrium structure at high temperatures. At lower temperatures the equilibrium phases are $\alpha-\mathrm{Cu}, T_{3}-\mathrm{Cu}_{3} \mathrm{Mn}_{2} \mathrm{Al}, \gamma_{2}-\mathrm{Cu}_{9} \mathrm{Al}_{4}$, and $\beta-\mathrm{Mn}$. When the metastable disordered bcc $\beta$ phase is retained on cooling, it successively undergoes two ordering transitions. ${ }^{10}$ In the first one, nearest-neighbor ordering is developed, and the $\beta_{2}$ phase appears; it has a $B 2$ ordered structure (space group, $P m 3 \mathrm{~m}$ ). In the second transition, next-nearest-neighbor ordering is developed and, depending on composition, the resulting phase is a solid solution based on the $\mathrm{DO}_{3}$ structure of $\mathrm{Cu}_{3} \mathrm{Al}$ ( $\beta_{1}$ phase) or the $L 2_{1}$ structure of the Heusler alloy $\mathrm{Cu}_{2} \mathrm{AlMn}\left(\beta_{3}\right.$ phase; in both $\beta_{1}$ and $\beta_{3}$ cases the space group is $F m 3 m$ ). Moreover, Bouchard and Thomas ${ }^{11}$ showed that, at lower temperatures (below $600 \mathrm{~K}$ ), a spinodal decomposition can occur between a nonmagnetic $\mathrm{Cu}_{3} \mathrm{Al}$-rich phase and a $\mathrm{Cu}_{2} \mathrm{AlMn}$-rich phase, which orders ferromagnetically. The kinetics of this process is slow and this decomposition is avoided for typical cooling rates. Associated with this spinodal decomposition some authors have reported the appearance of superparamagnetism at low temperatures. $^{12}$

The martensitic transformation takes place at a temperature that strongly depends on the composition, and also on other factors, such as the degree of atomic order of the parent phase, the presence of some amounts of precipitates of equilibrium phases, etc. ${ }^{13,14} \mathrm{In} \mathrm{Cu}-\mathrm{Al}-\mathrm{Mn}$ alloys, the formation of two different martensitic phases has been reported in the literature: ${ }^{15} \gamma^{\prime}$ phase, whose stacking sequence expressed in Ramsdell notation is $2 H$, develops when $e / a$ is larger than the $e / a$ value of the eutectoid point in the equilibrium phase diagram, ${ }^{16}$ which is 1.46 in $\mathrm{Cu}-\mathrm{Al}-\mathrm{Mn}$ (see Fig. 1). If $e / a$ is lesser than the value of the eutectoid point, $\beta^{\prime}$ phase is formed. This phase has an $18 R$ stacking sequence (if the parent phase is $\mathrm{DO}_{3}$ or $L 2_{1}$ ordered) or a $9 R$ one (if the parent phase is $B 2$ ordered or disordered). In other $\mathrm{Cu}$-based alloys, the same two martensitic phases appear above and below the composition of the eutectoid point. 


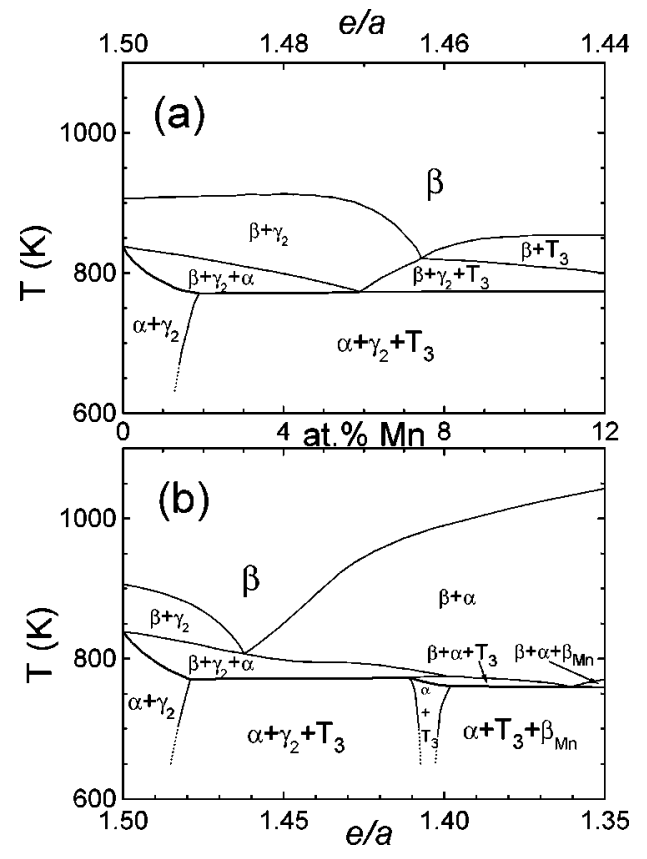

FIG. 1. Equilibrium phase diagram of $\mathrm{Cu}-\mathrm{Al}-\mathrm{Mn}$, taken from Ref. 9. (a) Along the composition line $\mathrm{Cu}_{3} \mathrm{Al} \rightarrow \mathrm{Mn}$. (b) Along the composition line $\mathrm{Cu}_{3} \mathrm{Al} \rightarrow \mathrm{Cu}_{3} \mathrm{Mn}_{2}$.

The present article is devoted to the study of the orderdisorder transitions in the Cu-Al-Mn system. The order and properties of these transitions have not been analyzed in detail in previous articles dealing with this subject and, moreover, contradictory informations about the order-disorder transitions in $\mathrm{Cu}_{3} \mathrm{Al}$ can be found in the literature. Due to both the fundamental and technological interests of these shape-memory alloys, this is a gap that must be filled, since order-disorder transitions have an influence over the stability of the bcc phase and over the martensitic transformation. On the other side, it is interesting from a fundamental point of view to find out whether the phase diagram of ternary alloys can be reproduced from simple theoretical hypotheses. This should provide physical information additional to that given by the experiments and, moreover, may be a tool for exploring regions of the phase diagram which can be difficult to reach by experiments. In this article, the order-disorder transitions of $\mathrm{Cu}-\mathrm{Al}-\mathrm{Mn}$ are tried to account for by means of Monte Carlo simulations on a simple proposed model for the system.

The paper is organized as it follows. In Sec. II the experimental details are explained. In Sec. III the experimental results concerning the order-disorder transitions are analyzed. In Sec. IV we introduce the simplified Blume-EmeryGriffiths model used in the simulations and discuss the results obtained from these simulations. In Sec. V the metastable phase diagram of $\mathrm{Cu}-\mathrm{Al}-\mathrm{Mn}$ is presented and in Sec. VI the main results and conclusions are summarized.

\section{EXPERIMENTAL DETAILS}

Two sets of composition related $\mathrm{Cu}-\mathrm{Al}-\mathrm{Mn}$ alloys were used for the experiments. The alloys of the first set belonged to the $\left(\mathrm{Cu}_{0.75} \mathrm{Al}_{0.25}\right)_{1-x} \mathrm{Mn}_{x}$ line, being $0 \leqslant x \leqslant 0.09$ for all the samples studied. The second set lay along the $\mathrm{Cu}_{3} \mathrm{Al}$
TABLE I. Atomic composition and average number of valence electrons per atom of the different alloys investigated. The first set of alloys belongs to the $\mathrm{Cu}_{3} \mathrm{Al} \rightarrow \mathrm{Mn}$ line and the second one to the $\mathrm{Cu}_{3} \mathrm{Al} \rightarrow \mathrm{Cu}_{3} \mathrm{Mn}_{2}$ line.

\begin{tabular}{lccc}
\hline \hline Alloy & at. \% $\mathrm{Al}$ & at. \% Mn & $e / a$ \\
\hline $\mathrm{Cu}_{3} \mathrm{Al}$ & 24.7 & 0.0 & 1.494 \\
$\mathrm{~A} 1$ & 24.9 & 5.0 & 1.499 \\
$\mathrm{~A} 2$ & 23.6 & 6.3 & 1.472 \\
$\mathrm{~A} 3$ & 23.8 & 6.9 & 1.475 \\
$\mathrm{~A} 4$ & 22.9 & 7.9 & 1.458 \\
$\mathrm{~A} 5$ & 22.8 & 9.0 & 1.456 \\
$\mathrm{~B} 00$ & & 5.3 & 1.437 \\
$\mathrm{~B} 05$ & 21.9 & 6.2 & 1.430 \\
$\mathrm{~B} 1$ & 21.5 & 7.0 & 1.435 \\
$\mathrm{~B} 15$ & 21.7 & 8.2 & 1.405 \\
$\mathrm{~B} 2$ & 20.3 & 9.1 & 1.399 \\
$\mathrm{~B} 3$ & 20.0 & 10.8 & 1.375 \\
\hline \hline
\end{tabular}

$\rightarrow \mathrm{Cu}_{3} \mathrm{Mn}_{2}$ line, i.e., the composition was $\mathrm{Cu}_{3} \mathrm{Al}_{1-x} \mathrm{Mn}_{2 x}$, with $0 \leqslant x \leqslant 0.22$ for all the alloys investigated. The atomic compositions for all the samples used are listed in Table I.

Polycrystalline cylindrical shaped ingots $(5 \mathrm{~mm}$ diameter) were obtained by melting pure elements $(99.99 \%)$. The samples used in the experiments were slices ( $2 \mathrm{~mm}$ thick) cut with a low-speed diamond saw. After being cut, each specimen was submerged into a $40 \% \mathrm{HNO}_{3}$ solution, in order to eliminate the damaged surface layer.

Calorimetric measurements corresponding to the $L 2_{1} \rightleftharpoons B 2$ transition were carried out in a commercial differential scanning calorimeter (DSC) from TA Instruments. In order to detect the $B 2 \rightleftharpoons A 2$ transition, which for some of the alloys occurs at temperatures above the upper limit of operation of our DSC $(870 \mathrm{~K})$, a high temperature differential thermal analysis (DTA) cell was used.

Before the calorimetric measurements of the orderdisorder transitions, samples were heat treated at $1080 \mathrm{~K}$ for $900 \mathrm{~s}$ and then quenched into an ice-water mixture, in order to prevent the precipitation of equilibrium phases. The quench was followed by a room-temperature annealing for more than $72 \mathrm{~h}$. For some specific compositions, we checked the effect of the thermal treatment by repeating the calorimetric experiments with samples that had been air cooled instead of quenched. All measurements were repeated in order to guarantee their reproducibility. The calorimetric runs were performed in the following way: from room temperature, samples were ballistically heated up to 570 or $620 \mathrm{~K}$ (depending on the specimen composition). Such fast heatings were carried out in order to minimize the time spent by the samples at a temperature range where equilibrium phases can grow. Next, data were acquired at a rate of $1 \mathrm{~Hz}$ as the specimens were heated up to $860 \mathrm{~K}$ at $10 \mathrm{~K} / \mathrm{min}$, and cooled down to $570 \mathrm{~K}$ again at the same temperature rate. In the end, they were fastly cooled down to room temperature. The same process was used for DTA measurements, but in this case the specimens were heated up to $1170 \mathrm{~K}$.

Powdered specimens for $\mathrm{x}$-ray diffraction measurements were obtained using a diamond file. The experiments were performed in a Bragg-Brentano diffractometer equipped with 
a high-temperature attachment and a position sensitive detector. The heating element was a Pt strip, and the temperature was measured with a Pt-10\% RhPt thermocouple placed on the strip. The powder was adhered to the heating element by means of a lacquer. High-temperature measurements of the order-disorder transitions were performed in a primaryvacuum atmosphere in order to avoid oxidation of the powder particles. After a preliminary study, we determined that a suitable procedure to study those transitions was the following: the powder, partially amorphized as a result of the filing, was annealed for one hour at $373 \mathrm{~K}$, for another hour at 473 $\mathrm{K}$, and for a third hour at $573 \mathrm{~K}$. Later, it was heated from this temperature up to $1123 \mathrm{~K}$ at steps of $30 \mathrm{~K}$, stopping for $180 \mathrm{~s}$ between step and step, in order to make two shortrange scans at $24^{\circ}<2 \theta<32^{\circ}$ and $40^{\circ}<2 \theta<48^{\circ}$. These positions covered the main superstructure and fundamental peaks of the $\beta_{1}$ phase and the main peaks characterizing the equilibrium phases. The heating rate at each step was $2 \mathrm{~K} / \mathrm{s}$. It was not possible to perform equivalent measurements on cooling because precipitation of equilibrium phases occurred, which interfered strongly with the two ordering transitions.

\section{ORDER-DISORDER TRANSITIONS}

\section{A. Experimental results}

Depending on the composition of the samples, one or two phase transitions were detected by high-temperature calorimetric measurements. The transition that occurs at lower temperature gives rise to a high calorimetric peak, and was observed in all the samples studied. A second transition occurs at higher temperatures, which was not detected for all the samples. It gives rise to a weak calorimetric peak, with an area which is always less than one tenth of the area of the peak corresponding to the first transition.

In order to corroborate that the peaks observed in the thermal curves are associated with the configurational ordering or disordering phase transitions of the samples studied, $\mathrm{x}$-ray diffraction measurements with powders of specimen A4 were performed, heating from room temperature. This alloy was chosen because it has a composition close to the eutectoid point and, also, because it is one of the specimens with highest transition temperatures; therefore, less problems arising from the precipitation of equilibrium phases were expected. The results obtained are plotted in Fig. 2(a), which shows the relative integrated intensities of the (111) and (200) superstructure peaks. These peaks correspond respectively to the symmetry of the $\mathrm{L2}_{1}$ (or $\mathrm{DO}_{3}$ ) ordered phase and the symmetry of the $B 2$ ordered phase (see Appendix A). Figure 2(b) displays the heating thermal curve of a sample with the same composition, measured by DSC. The thermal curve obtained by DTA for the same sample is plotted in Fig. 2(c). Though the DTA cell is less sensitive and yields a much worse baseline, this measurement confirms that there is a phase transition at $\sim 858 \mathrm{~K}$, which was not finished at the end of the DSC experiment [Fig. 2(b)]. The $\mathrm{x}$-ray results show that this is the $B 2 \rightarrow A 2$ transition, and also that the larger calorimetric peak below $800 \mathrm{~K}$ corresponds to the $L 2_{1} \rightarrow B 2$ (or $\mathrm{DO}_{3} \rightarrow B 2$ ) transition.

Since the $\mathrm{X}$-ray scattering factors for $\mathrm{Cu}$ and for $\mathrm{Mn}$ are very similar, there are no appreciable differences in the ex-

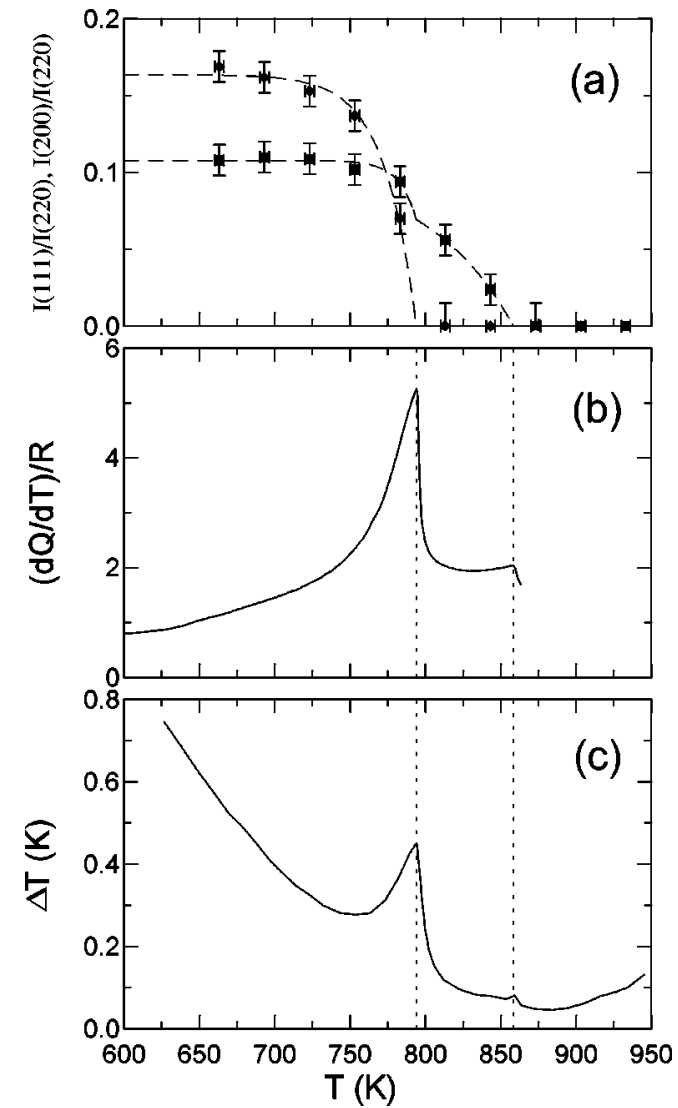

FIG. 2. (a) Relative integrated intensity of the (111) ( ) and (200) (ם) superstructure x-ray diffraction peaks for powders of sample A4. Dashed lines are fits corresponding to a mean-field critical exponent $\beta=1 / 2$ near the critical point. (b) Thermogram recorded by a DSC experiment on heating a sample of identical composition, and (c) thermogram obtained by DTA on the same sample. $R$ is the ideal gas constant, $R=8.314 \mathrm{~J} / \mathrm{mol} \mathrm{K}$.

pected diffraction-peak intensities between a $\mathrm{DO}_{3}$ ordered lattice and an $L 2{ }_{1}$ ordered lattice, and we cannot distinguish between these two types of ordering of the lattice from our x-ray measurements. A study about which of these two ordered phases appear, from transmission electron microscopy (TEM) and electron diffraction data, was performed in Refs. 11 and 14, which showed that there is $\mathrm{DO}_{3}$ ordering at low concentrations of $\mathrm{Mn}$ and $L 2_{1}$ ordering at higher Mn contents. For the sake of simplicity, we will refer hereafter to $L 2{ }_{1}$ order for all $\mathrm{Cu}-\mathrm{Al}-\mathrm{Mn}$ alloys, and we will consider explicitly the $\mathrm{DO}_{3}$ phase only in the case of $\mathrm{Cu}_{3} \mathrm{Al}$.

For all the alloys studied, the temperatures of the orderdisorder transitions were obtained from the position of the peaks in the DSC and DTA thermal curves. Some of these curves are shown in Fig. 3. For $\mathrm{Cu}_{3} \mathrm{Al}$ [Fig. 3(b)], not all the peaks appearing in the thermal curve are associated with order-disorder transitions: a detailed discussion on the origin of each of the peaks observed for this sample will be held in next subsection. The complete set of order-disorder transition temperatures obtained for all the samples is listed in Table II, and plotted in Fig. 4 as a function of the atomic Mn content.

The reproducibility of the experiments was checked, and the values listed in Table II were found to be reproducible within a margin of $\pm 1 \mathrm{~K}$, except for measurements on 


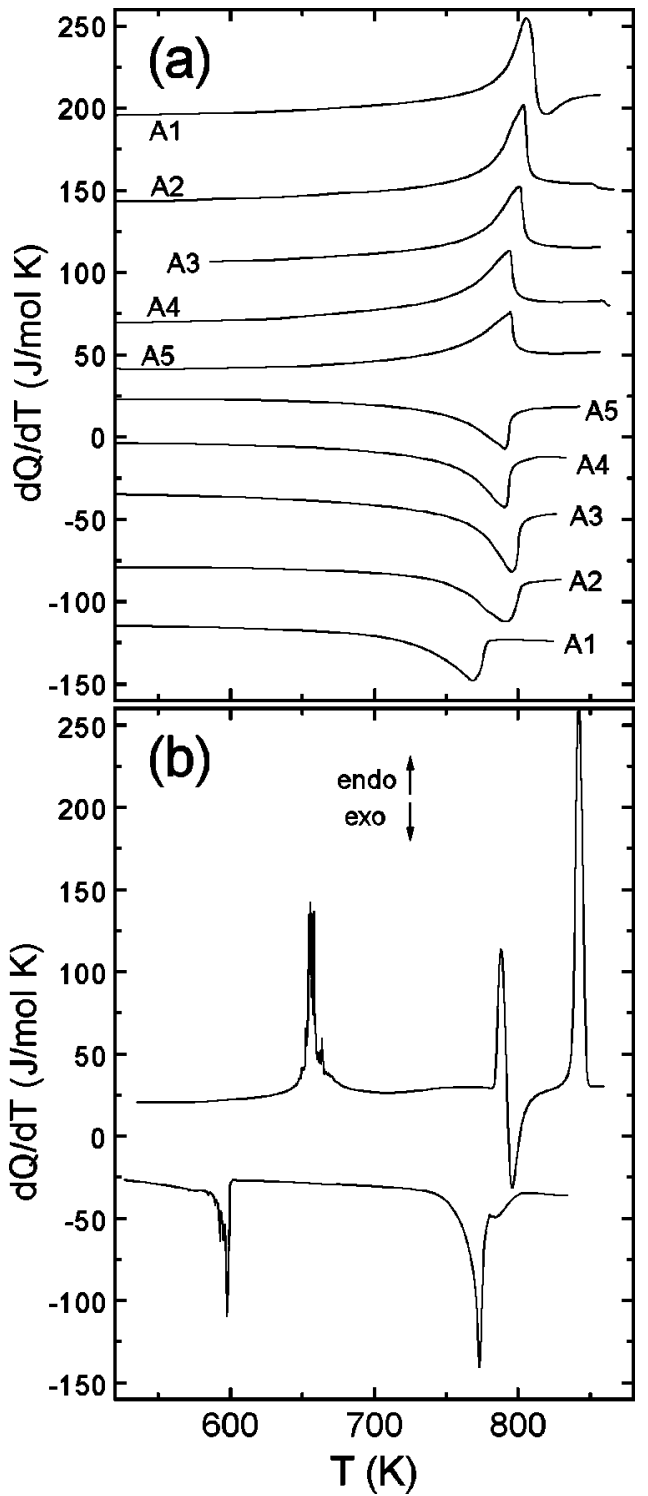

FIG. 3. (a) DSC thermal curves corresponding to the orderdisorder transitions of the set of $\mathrm{Cu}-\mathrm{Al}-\mathrm{Mn}$ samples belonging to the $\mathrm{Cu}_{3} \mathrm{Al} \rightarrow \mathrm{Mn}$ line. (b) DSC thermal curves in the same temperature range for an as-quenched $\mathrm{Cu}_{3} \mathrm{Al}$ sample. In the two figures, plots are shifted vertically to improve clarity.

samples B15, B2, and B3, which were affected by a larger scatter (up to $\pm 7 \mathrm{~K}$ ), arising from a certain degradation of the shape of the thermal peak. This fact was probably due to the interplay between the order-disorder transitions and some amount of precipitated equilibrium phases, since the compositions of these three samples are far from the eutectoid point.

The effect of the heating rate on the DSC measurements of the $L 2_{1} \rightleftharpoons B 2$ transition was investigated for samples A4 and $\mathrm{B} 05$, in the $d T / d t$ range from 1 to $20 \mathrm{~K} / \mathrm{min}$. The temperature and height of the maximum of the thermal peak obtained on heating showed no dependence with $d T / d t$. In contrast, the temperature of the maximum obtained on cooling was reduced as the cooling rate was increased. The reason for this behavior arises from the finite kinetic response of the experimental device: since the thermal curve $d Q / d T$ has a very large slope in the range of temperatures of some de-
TABLE II. $L 22_{1} \rightleftharpoons B 2$ and $B 2 \rightleftharpoons A 2$ transition temperatures of $\mathrm{Cu}-\mathrm{Al}-\mathrm{Mn}$, measured on heating $\left(T^{h}\right)$ and on cooling $\left(T^{c}\right)$, respectively.

\begin{tabular}{lcccc}
\hline \hline Alloy & $T_{L 2_{1} \rightarrow B 2}^{h}(\mathrm{~K})$ & $T_{B 2 \rightarrow L 2_{1}}^{c}(\mathrm{~K})$ & $T_{B 2 \rightarrow A 2}^{h}(\mathrm{~K})$ & $T_{A 2 \rightarrow B 2}^{c}(\mathrm{~K})$ \\
\hline $\mathrm{Cu}_{3} \mathrm{Al}$ & 788 & 776 & 788 & 776 \\
$\mathrm{~A} 1$ & 806 & 779 & & \\
$\mathrm{~A} 2$ & 803 & 802 & 848 & \\
$\mathrm{~A} 3$ & 801 & 801 & 858 & 859 \\
$\mathrm{~A} 4$ & 794 & 795 & 858 & 858 \\
$\mathrm{~A} 5$ & 795 & 795 & 870 & 869 \\
$\mathrm{~B} 00$ & 776 & 769 & & \\
$\mathrm{~B} 05$ & 772 & 771 & & \\
$\mathrm{~B} 1$ & 769 & 760 & & \\
$\mathrm{~B} 15$ & 760 & 741 & & 816 \\
$\mathrm{~B} 2$ & 759 & 722 & 824 & 821 \\
$\mathrm{~B} 3$ & 756 & 740 & 846 & 826 \\
\hline \hline
\end{tabular}

grees above the maximum of the transition [see Fig. 3(a)], the kinetic response of the apparatus is not fast enough in this range, and this causes a delay in the position of the maximum of the thermal peak on cooling. In this case, the temperature of the onset point (i.e., the intersection between the base line and the tangent to the thermal curve at the point of maximum slope) does not show any dependence with $d T / d t$, giving a more reliable value for the temperature of the $B 2 \rightarrow L 2_{1}$ transition. Therefore, the results presented in Table II correspond to the maximum of the thermal peak for the heating transitions and to the onset point for the cooling ones. Their estimated total error is $\pm 2 \mathrm{~K}$.

The dependence of the order-disorder transitions on the heat treatment performed on the sample was also investigated. DSC measurements on a sample of specimen A2 were

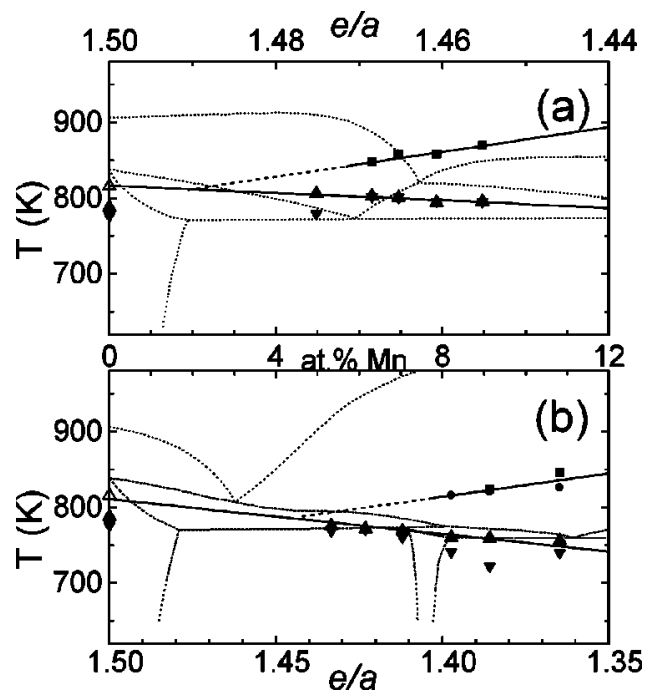

FIG. 4. Order-disorder transition temperatures of samples belonging to (a) the $\mathrm{Cu}_{3} \mathrm{Al} \rightarrow \mathrm{Mn}$ and (b) the $\mathrm{Cu}_{3} \mathrm{Al} \rightarrow \mathrm{Cu}_{3} \mathrm{Mn}_{2}$ alloy families. $\boldsymbol{\Delta}: L 2_{1} \rightarrow B 2$ and $L 2_{1}\left(D O_{3}\right) \rightarrow A 2$ transitions; $\boldsymbol{\nabla}: B 2$ $\rightarrow L 2_{1}$ and $A 2 \rightarrow L 2_{1}\left(D O_{3}\right) ; \mathbf{\square}: B 2 \rightarrow A 2 ; \mathbf{0}: A 2 \rightarrow B 2$. Solid symbols: present results. Open symbols: taken from Ref. 11. Solid and dashed lines are linear fits to the data. Dotted lines correspond to the equilibrium phase diagram of Fig. 1. 
carried out (i) $96 \mathrm{~h}$ after a quench from $1080 \mathrm{~K}$, (ii) just $\sim 300 \mathrm{~s}$ after an identical quench, and (iii) $96 \mathrm{~h}$ after an air cooling from the same temperature. Similar thermograms were obtained for the three experiments, with comparable base lines and thermal peaks at identical temperatures with identical heights and areas. This result shows that the state of the sample is similar, no matter how the previous heat treatment has been performed, and indicates that it is very difficult to retain an important degree of frozen disorder by quenching in $\mathrm{Cu}-\mathrm{Al}-\mathrm{Mn}$ alloys, contrarily to what has been observed in other $\mathrm{Cu}$-based alloys, such $\mathrm{Cu}-\mathrm{Zn}-\mathrm{Al},{ }^{17}$ which has lower $L 2_{1} \rightleftharpoons B 2$ transition temperatures. Actually, we have never been able to observe an appreciably disordered $\beta$-Cu-Al-Mn sample by $\mathrm{x}$-ray diffraction measurements at room temperature, regardless of which heat treatment was performed.

In addition to the order-disorder transition temperatures, the energy change at these transitions can also be evaluated from our measurements. This change is approximately equal to the enthalpy change, since the energetic contribution due to volume changes at the order-disorder transition is negligible in this kind of $\mathrm{Cu}$-based alloys: From x-ray diffraction results, we have evaluated the lattice parameter of the sample A4 as a function of temperature; it changes from $a=5.91$ $\AA$ at $600 \mathrm{~K}$ to $a=5.96 \AA$ at $1000 \mathrm{~K}$, and this leads to a volume change between these two temperatures (which are, respectively, lesser and higher than the order-disorder transition temperatures) $\Delta V \sim 2 \times 10^{-7} \mathrm{~m}^{3} / \mathrm{mol}^{18}$ Hence, the volume change renders a contribution to the enthalpy change $P \cdot \Delta V \sim 0.02 \mathrm{~J} / \mathrm{mol}$, which is completely negligible. Therefore, we can disregard this contribution and evaluate experimentally the internal energy change of the samples at the order-disorder transitions by simple integration of the calorimetric peak:

$$
\Delta E_{\mathrm{exp}}^{\mathrm{ord} \rightarrow \mathrm{dis}} \approx \Delta H_{\mathrm{exp}}^{\mathrm{ord} \rightarrow \mathrm{dis}}=\int_{T_{1}}^{T_{2}}\left[\frac{d Q}{d T}-\left(\frac{d Q}{d T}\right)_{\mathrm{BL}}\right] d T,
$$

where $T_{1}$ and $T_{2}$ are temperatures below and above the transition, respectively, and $(d Q / d T)_{\mathrm{BL}}$ is the base line, which contains all the thermodynamic contributions not arising from the configurational ordering of the sample.

The $\Delta E_{\mathrm{exp}}^{\text {ord }- \text { dis }}$ results computed at the $L 2_{1} \rightleftharpoons B 2$ transition are shown in Table III. The temperature $T_{1}$ is ill defined and it can be more than $100 \mathrm{~K}$ below the temperature of the calorimetric peak, causing a considerable error bar in the obtained results, ${ }^{19}$ as is also shown in Table III. It is worth noting that the energy change evaluated from this method does not contain the total energy difference between the ordered and the disordered phases $\Delta E_{\mathrm{tot}}^{\mathrm{ord} \rightarrow \text { dis }}$, which would be obtained in the theoretical situation $T_{1}=0$ (completely ordered sample) and $T_{2} \rightarrow \infty$ (completely disordered one). If, e.g., sample A4 is considered, the values $T_{1}=640 \mathrm{~K}$ and $T_{2}=830 \mathrm{~K}$ were taken for the integration. It can be observed in Fig. 2 that the alloy has practically reached the maximum order at $T_{1}$. At $T_{2}$, there is no remaining long-range $L 2_{1}$ order, but there is still short-range order, whose significative energetic contribution is not contained in our integration. It has also to be taken into account that a part of the contribution arising from the reduction of the $B 2$ order parameter must be contained in integral (1). But this contribution is
TABLE III. Measured energy changes in the $L 2_{1} \rightleftharpoons B 2$ transitions ( $L 2_{1} \rightleftharpoons A 2$ for low Mn contents), obtained on heating $\left(\Delta E^{h}\right)$ and on cooling $\left(\Delta E^{c}\right)$ the samples.

\begin{tabular}{lrc}
\hline \hline Alloy & \multicolumn{1}{c}{$\Delta E^{h}(\mathrm{~J} / \mathrm{mol})$} & $\Delta E^{c}(\mathrm{~J} / \mathrm{mol})$ \\
\hline A1 & $1260 \pm 250$ & $1020 \pm 220$ \\
A2 & $1160 \pm 90$ & $1190 \pm 300$ \\
A3 & $1120 \pm 150$ & $1160 \pm 200$ \\
A4 & $1060 \pm 200$ & $1100 \pm 160$ \\
A5 & $900 \pm 180$ & $1030 \pm 140$ \\
B00 & $1360 \pm 50$ & \\
B05 & $1310 \pm 60$ & \\
B1 & $1240 \pm 70$ & \\
B15 & $1080 \pm 80$ & \\
B2 & $920 \pm 110$ & \\
B3 & $700 \pm 200$ & \\
\hline \hline
\end{tabular}

small (of the order of the error bars), as it can be seen from the low height of the $B 2 \rightarrow A 2$ thermal peak in relation to the height of the $L 2_{1} \rightarrow B 2$ peak.

\section{B. Symmetry of the different phases and order of the transitions}

For both sets of alloys studied, the height of the $B 2 \rightleftharpoons A 2$ calorimetric peak decreases as the at. \% $\mathrm{Mn}$ is reduced, and its temperature drops, approaching the $L 2_{1} \rightleftharpoons B 2$ transition temperature, so that only one transition is detected for the lowest Mn concentrations. This finding is in agreement with the hypothesis that there is a single disorder-order transition in $\mathrm{Cu}_{3} \mathrm{Al}$, from the disordered $\mathrm{A} 2$ (bcc) phase to the $\mathrm{DO}_{3}$ structure, and indeed only one transition has been detected in our calorimetric measurements, as it will be discussed later. Actually, a single $\mathrm{DO}_{3} \rightleftharpoons A 2$ transition has been reported in a number of phase diagrams of $\mathrm{Cu}-\mathrm{Al}$ (Ref. 20) and also in recent published measurements on order-disorder transitions in $\mathrm{Cu}_{3} \mathrm{Al}(\mathrm{Be}) ;{ }^{21}$ in addition, published results for $\mathrm{Cu}-\mathrm{Zn}-\mathrm{Al}^{22}$ show that, as in $\mathrm{Cu}-\mathrm{Al}-\mathrm{Mn}$, both $B 2 \rightleftharpoons A 2$ and $L 2_{1} \rightleftharpoons B 2$ transitions join in a single transition on reducing the $\mathrm{Zn}$ content down to 0 . Nevertheless, $\mathrm{x}$-ray diffraction results have been published ${ }^{10}$ showing two order-disorder transitions in $\mathrm{Cu}_{3} \mathrm{Al}$. As it was discussed in detail in Ref. 21, this discrepancy could be due to the growth of the $\gamma_{2}$ equilibrium phase, one of whose x-ray peaks was taken as the (200) $B 2$ superstructure peak. Also, resistivity measurements on $\mathrm{Cu}-\mathrm{Al}-\mathrm{Mn}$ by Prado et al. ${ }^{23}$ give high-temperature values for the $B 2 \rightleftharpoons A 2$ transition of two samples with low Mn content $(<4$ at. \%), but these findings are not confirmed by other experimental techniques. ${ }^{11}$ As far as we know, the measurements of Prado are the only published values in agreement with the results from Ref. 10; nevertheless, for higher Mn contents (where the $\beta$ phase is more stable and the $B 2 \rightleftharpoons A 2$ transition is more easily detected) Prado's results agree with those presented in this paper.

Next, we would like to discuss the order of the orderdisorder transitions in $\mathrm{Cu}-\mathrm{Al}-\mathrm{Mn}$. It can be observed [see Fig. 3(a), samples A2-A5] that the calorimetric $L 2_{1} \rightleftharpoons B 2$ peaks of $\mathrm{Cu}-\mathrm{Al}-\mathrm{Mn}$ are broad, spreading down to temperatures more than $100-150 \mathrm{~K}$ below the position of the maxi- 
mum; they have the characteristic lambda shape expected for a continuous transition. The smaller $B 2 \rightarrow A 2$ peaks also seem to have a lambda shape, superimposed on the decreasing high-temperature tail of the $L 2_{1} \rightarrow B 2$ peaks. Moreover, within the experimental uncertainties, no hysteresis is detected for these transitions (except for the samples with lowest e/ $a$ values; we will discuss this subject later on). The $\mathrm{X}$-ray diffraction results from sample A4 (Fig. 2) are in agreement with a second order transition too: the long-range order parameters decrease gradually from temperatures far below the transition temperature, and vanish at the critical point, where there does not seem to be a jump in their value.

Therefore, we conclude that two second order orderdisorder transitions occur in $\mathrm{Cu}-\mathrm{Al}-\mathrm{Mn}$ with $\mathrm{Mn}$ concentration above 5 at. $\%: L 2_{1} \rightleftharpoons B 2$ and $B 2 \rightleftharpoons A 2$. The fact that these two transitions are second order is in agreement with symmetry arguments based on Landau theory (see Appendix A). From these arguments we can also state that, in the range of compositions where these two continuous transitions occur, the low temperature phase must be $L 2_{1}$ and not $D O_{3}$.

Let us analyze now the thermal curves obtained for $\mathrm{Cu}_{3} \mathrm{Al}$ [Fig. 3(b)], which look very different from the curves obtained for the ternary $\mathrm{Cu}-\mathrm{Al}-\mathrm{Mn}$ samples. Contrarily to what is observed for those ternary alloys, not all the peaks displayed in the figure for $\mathrm{Cu}_{3} \mathrm{Al}$ are related to order-disorder transitions. The first peak, around $600 \mathrm{~K}$, is associated with the martensitic transformation, which occurs at higher temperatures than for $\mathrm{Cu}-\mathrm{Al}-\mathrm{Mn}$ alloys. Later on heating, a first order transition is observed at $788 \mathrm{~K}$ : this is the previously discussed order-disorder $\mathrm{DO}_{3} \rightarrow A 2$ transition. Immediately after the beginning of this transition, an exothermal peak is superimposed on the endothermal one related to the orderdisorder transition. The exothermal peak is due to the decomposition of the bcc phase into the equilibrium $\alpha$ and $\gamma_{2}$ phases. This precipitation following the disordering of the alloy is also observed, though in a lesser degree, in sample A1 (see Fig. 3); ${ }^{24}$ it is an evidence of the higher stability to decomposition of the ordered phase than of the disordered one.

The largest endothermal peak observed on heating in $\mathrm{Cu}_{3} \mathrm{Al}$ is due to the eutectoid reaction $\alpha+\gamma_{2} \rightarrow \beta$ at $838 \mathrm{~K} ;{ }^{20}$ after this reaction the bcc phase is recovered. On cooling, the $A 2 \rightarrow \mathrm{DO}_{3}$ transition occurs at $776 \mathrm{~K}$; the hysteresis of the transition is $\sim 12 \mathrm{~K}$. The precipitation of equilibrium phases is a slow process that strongly depends on the cooling rate. At the rate used in our experiment $(10 \mathrm{~K} / \mathrm{min})$ it is not completely avoided, as evidenced by the depression of the base line previous to the $A 2 \rightarrow \mathrm{DO}_{3}$ transition. Because of this partial precipitation, the thermal peak related to the martensitic transformation is slightly smaller on cooling than on heating (prior to the experiment the sample had been quenched so that precipitation had been avoided).

We have seen that $\mathrm{Cu}_{3} \mathrm{Al}$ displays a single first order $\mathrm{DO}_{3} \rightleftharpoons A 2$ transition, while two second order transitions, $L 2_{1} \rightleftharpoons B 2$ and $B 2 \rightleftharpoons A 2$, are present in Cu-Al-Mn with more than 5 at. $\% \mathrm{Mn}$. These two phase transitions must join at some point of the phase diagram, at lesser Mn concentrations, but it is not possible to establish the exact position of this point from the results presented in this paper. Though only one transition has been found for sample A1, the $B 2$ $\rightarrow A 2$ transition could have been hidden in this alloy by the precipitating reaction, which could also be responsible for the hysteresis observed in the order-disorder transition.

Precipitation can also account for the thermal hysteresis in the $L 2_{1} \rightleftharpoons B 2$ transition exhibited by the samples with higher $\mathrm{Mn}$ content of the $\mathrm{Cu}_{3} \mathrm{Al} \rightarrow \mathrm{Cu}_{3} \mathrm{Mn}_{2}$ line. These specimens have compositions far from the eutectoid point, which result in a competition between the order-disorder transition and the precipitation of equilibrium phases; such a competition could modify the properties of the order-disorder transition. Actually, the shape of the thermograms corresponding to the order-disorder transitions of these samples was difficult to reproduce, contrarily to what happened for the rest of specimens. These problems arose from precipitation too, and a detailed study of this effect is beyond the scope of this paper.

\section{MONTE CARLO SIMULATIONS}

In the previous section it has been shown that, experimentally, two continuous order-disorder transitions are found for bcc $\mathrm{Cu}-\mathrm{Al}-\mathrm{Mn}$ alloys with high $\mathrm{Mn}$ contents, and these two transitions join in a single first order one on reducing the $\mathrm{Mn}$ concentration. We are interested now in finding if this scenario can be reproduced by means of simple theoretical models based on a reduced number of hypotheses. This can provide enlightening on the physical reasons why one or two transitions exist depending on composition and can give additional information about the distribution of the different atomic species among the different sublattices of the crystal. In order to satisfy our aim, a possibility is to model the interactions among the atoms of the crystal lattice and to perform equilibrium Monte Carlo simulations ${ }^{25}$ on this lattice. This method has been widely and successfully used in the last years for the study of order-disorder transitions on binary alloys, and we expect that it can also be useful to meet our demands for the ternary alloy studied in this article.

For the interactions among the atoms of the alloy, we have used the Blume-Emery-Griffiths ${ }^{26}$ Hamiltonian, which is equivalent to a pair interaction lattice model (see Appen$\operatorname{dix}$ B). The fact that there are three possible states for the pseudospin variable makes this Hamiltonian a natural choice for ternary alloys. Actually, it has recently been used in other works. $^{27}$ It reads as

$$
\begin{aligned}
H= & \sum_{\langle i j\rangle}^{\mathrm{NN}}\left\{J_{1} S_{i} S_{j}+K_{1} S_{i}^{2} S_{j}^{2}+L_{1}\left(S_{i} S_{j}^{2}+S_{i}^{2} S_{j}\right)\right\} \\
& +\sum_{\langle i j\rangle}^{\mathrm{NNN}}\left\{J_{2} S_{i} S_{j}+K_{2} S_{i}^{2} S_{j}^{2}+L_{2}\left(S_{i} S_{j}^{2}+S_{i}^{2} S_{j}\right)\right\},
\end{aligned}
$$

where $S_{i}=1,0,-1$ when the $i$ site is occupied by a $\mathrm{Cu}, \mathrm{Mn}$, or $\mathrm{Al}$ atom, respectively. The sum $\Sigma_{\langle i j\rangle} \mathrm{NN}$ is extended over all the nearest neighbor (NN) pairs and the sum $\Sigma_{\langle i j\rangle} \mathrm{NNN}$ over all the next-nearest-neighbor (NNN) pairs. It is not necessary to consider interactions among atoms beyond nextnearest neighbors to obtain the ordered phases in which we are interested.

In a first stage, prior to the simulations, we analyzed the ground states of $\mathrm{Cu}_{2} \mathrm{AlMn}$ and $\mathrm{Cu}_{3} \mathrm{Al}$ for different values of the six parameters of the complete Blume-Emery-Griffiths model. We found that the experimental ground states $\left(L 2_{1}\right.$ for $\mathrm{Cu}_{2} \mathrm{AlMn}$ and $\mathrm{DO}_{3}$ for $\mathrm{Cu}_{3} \mathrm{Al}$ ) could be reproduced with 


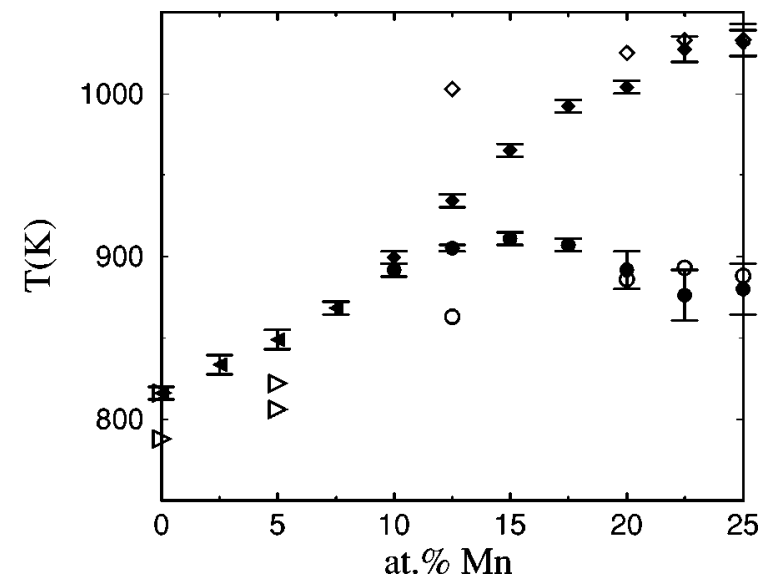

FIG. 5. Order-disorder transitions along the $\mathrm{Cu}_{3} \mathrm{Al} \rightarrow \mathrm{Cu}_{2} \mathrm{AlMn}$ line, measured experimentally (open symbols, taken from Ref. 11 and this work), and computed from Monte Carlo simulations on a lattice with $2 \times 64^{3}$ atoms (solid symbols). Triangles: first order $L 2_{1}\left(D O_{3}\right) \rightleftharpoons A 2$ transitions. Circles: second order $L 2_{1} \rightleftharpoons B 2$ transitions. Diamonds: second order $B 2 \rightleftharpoons A 2$ transitions.

a Hamiltonian containing only the $J_{1}, J_{2}$, and $L_{2}$ terms. Therefore, in order to avoid a model having an excessive number of adjustable parameters, we truncated the full Hamiltonian by taking the parameters $L_{1}, K_{1}$, and $K_{2}$ to be zero, and considered this simpler three-parameter version of the Blume-Emery-Griffiths model in all the subsequent simulations.

In a second stage, we performed simulations on $\mathrm{Cu}_{3} \mathrm{Al}$ and $\mathrm{Cu}_{2}$ AlMn for different values of $J_{2} / J_{1}$ and $L_{2} / J_{1}$. We obtained a range of values of these parameters for which a single first order $\mathrm{DO}_{3} \rightleftharpoons A 2$ transition was observed for $\mathrm{Cu}_{3} \mathrm{Al}$ and two second order transitions, $L 2_{1} \rightleftharpoons B 2$ and $B 2 \rightleftharpoons A 2$, appeared for $\mathrm{Cu}_{2} \mathrm{AlMn}$, in complete agreement with the experimental observations. Further simulations on intermediate compositions allowed us to find the existence of a point where the two continuous transitions joined in a single first order one; the position of this point in the phase diagram depended on the values of $J_{2} / J_{1}$ and $L_{2} / J_{1}$.

In order to complete the simulations, we fixed the values of $J_{2} / J_{1}$ and $L_{2} / J_{1}$ in such a way that they reproduce the following experimental data from Ref. 11: $T_{2} / T_{3} \simeq 0.86$ and $T_{3} / T_{1} \simeq 1.27$, where $T_{1}$ is the temperature of the $D O_{3} \rightleftharpoons A 2$ transition of $\mathrm{Cu}_{3} \mathrm{Al}$, and $T_{2}$ and $T_{3}$ are the temperatures of the $L 2_{1} \rightleftharpoons B 2$ and $B 2 \rightleftharpoons A 2$ transitions of $\mathrm{Cu}_{2} \mathrm{AlMn}$, respectively. These conditions were satisfied taking $J_{2} / J_{1}=0.65$ and $L_{2} / J_{1}=-0.55$. Finally, the value of $J_{1}$ fixes the scale of temperatures and energies, so that a direct comparison between experimental and simulation results is possible. Adjusting the temperature $T_{1}$ given by our simulations to the experimental one, ${ }^{11} T_{1} \simeq 816 \mathrm{~K}$, we have $J_{1} / k_{B} \simeq 388 \mathrm{~K}$.

The phase diagram obtained with these parameters for the $\mathrm{Cu}_{3-x} \mathrm{AlMn}_{x}$ line (alloys with 25 at. \% $\mathrm{Al}$ ) is presented in Fig. 5. From the simulations, a single first order $L 2_{1} \rightleftharpoons A 2$ transition is found for $x \leqq 0.4$ ( $\sim 10$ at. \% Mn), while there are two continuous order-disorder transitions, $L 2_{1} \rightleftharpoons B 2$ and $B 2 \rightleftharpoons A 2$, for $x \gtrsim 0.4$.

These results corroborate the experimental data obtained in Ref. 11 and in the present work. ${ }^{28}$ Nevertheless, the compositions of the alloys studied in the present article do not

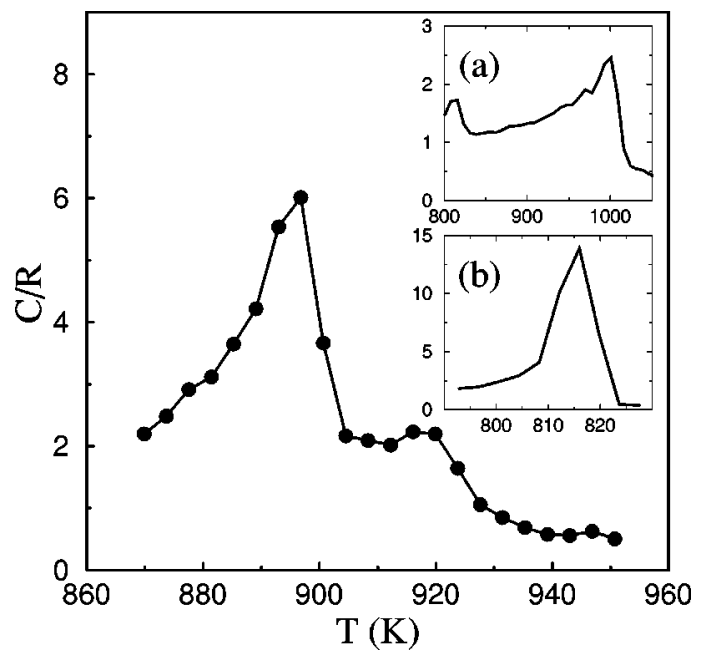

FIG. 6. Configurational heat capacity $C$ of $\mathrm{Cu}_{2.5} \mathrm{AlMn}_{0.5}$, computed from Monte Carlo simulations performed on heating. Insets: $C$ of (a) $\mathrm{Cu}_{2} \mathrm{AlMn}$ and (b) $\mathrm{Cu}_{3} \mathrm{Al}$, computed by the same method. All simulations have been carried out on a lattice with $2 \times 64^{3}$ atoms. Notice the different horizontal and vertical scales for each composition.

exactly fall on the $\mathrm{Cu}_{3-x} \mathrm{AlMn}_{x}$ line (though they are close to it). Hence, with the same parameters, we have explicitly simulated systems with the compositions of the A series of Table I. We have obtained transition temperatures similar to those measured experimentally, but the $L 2_{1} \rightleftharpoons A 2$ transition splits into two transitions at higher Mn concentrations in the simulations ( $\sim 8$ at. $\% \mathrm{Mn})$ than the concentration at which the splitting is observed experimentally ( $\sim 5$ at. $\% \mathrm{Mn})$. Therefore, our simulations overestimate the Mn concentration value at which the splitting from one into two transitions occurs: this also explains the discrepancy between experimental and simulation data at 12.5 at. \% Mn (see Fig. 5). The reason for the bad estimation of the splitting point could arise from the simplicity of the model used, since we have considered only three of the six possible parameters in the full Blume-Emery-Griffiths Hamiltonian and we have not taken into account that these parameters may, on their turn, be composition dependent.

The heat capacity obtained from simulations of the $\mathrm{Cu}_{2.5} \mathrm{AlMn}_{0.5}$ alloy is shown in Fig. 6. It can be seen that it has a shape very similar to that observed in the thermogram of Fig. 2(b), where the peak associated with the $L 2_{1} \rightleftharpoons B 2$ transition is much more pronounced than the peak associated with the $B 2 \rightleftharpoons A 2$ transition. The heat capacity at the left (i.e., lower temperatures) of this second peak has, in both experimental and simulation cases, very little slope. Figure 6 also shows the heat capacity for $\mathrm{Cu}_{2} \mathrm{AlMn}$ and $\mathrm{Cu}_{3} \mathrm{Al}$ alloys. It can be seen that in the first case the peak corresponding to the $B 2 \rightleftharpoons A 2$ transition is higher than the $L 2_{1} \rightleftharpoons B 2$ peak, contrarily to what occurs at lower Mn compositions. Besides, in the $\mathrm{Cu}_{3} \mathrm{Al}$ case, a single peak of the heat capacity is observed. This peak is more pronounced, as corresponds to a first-order transition. In this case, we have also simulated the system on cooling, and we have found an hysteresis of $\sim 12$ $\mathrm{K}$, in excellent agreement with our experimental result. The energy change at the $\mathrm{DO}_{3} \rightleftharpoons A 2$ transition obtained in the simulations is $\Delta E \simeq 900 \mathrm{~J} / \mathrm{mol}$ on heating and $\Delta E \simeq 1160 \mathrm{~J} /$ 
mol on cooling, which are values of the same order of magnitude as those shown in Table III.

With the values obtained for $J_{1}, J_{2}$, and $L_{2}$, we can calculate the exchange energies (see Appendix B) for $\mathrm{Cu}-\mathrm{Al}$ $\mathrm{Mn}$. These energies are

$$
\begin{aligned}
& \frac{J_{\mathrm{CuAl}}^{\mathrm{NN}}}{k_{B}}=1552 \mathrm{~K}, \\
& \frac{J_{\mathrm{CuAl}}^{\mathrm{NNN}}}{k_{B}}=1008 \mathrm{~K}, \\
& \frac{J_{\mathrm{CuMn}}^{\mathrm{NN}}}{k_{B}}=388 \mathrm{~K}, \\
& \frac{J_{\mathrm{CuMn}}^{\mathrm{NNN}}}{k_{B}}=-175 \mathrm{~K}, \\
& \frac{J_{\mathrm{AlMn}}^{\mathrm{NN}}}{k_{B}}=388 \mathrm{~K}, \\
& \frac{J_{\mathrm{AlMn}}^{\mathrm{NNN}}}{k_{B}}=679 \mathrm{~K} .
\end{aligned}
$$

The conditions $L_{1}=0$ and $K_{1}=0$ fix the quantities $J_{\text {CuMn }}^{\mathrm{NN}}$ and $J_{\mathrm{AlMn}}^{\mathrm{NN}}$ from $J_{\mathrm{CuAl}}^{\mathrm{NN}}$, and the condition $K_{2}=0$ establishes a link between $J_{\mathrm{CuMn}}^{\mathrm{NNN}}$ and $J_{\mathrm{AlMn}}^{\mathrm{NNN}}$. Therefore, it will be difficult to compare the $\mathrm{Cu}-\mathrm{Mn}$ and $\mathrm{Al}-\mathrm{Mn}$ exchange energies presented here with values obtained by other methods. In spite of this, the significant similarities between the experimental and simulated phase diagrams seem to show that the energies calculated in Eq. (3) may be realistic.

It is remarkable to notice that the values presented here for $J_{\mathrm{CuAl}}^{\mathrm{NN}}$ and $J_{\mathrm{CuAl}}^{\mathrm{NNN}}$ are in good agreement with values published previously for different $\mathrm{Cu}$-based alloys, obtained by means of a Bragg-Williams model modified in order to account for short-range ordering: Ref. 22 gives $J_{\mathrm{CuAl}}^{\mathrm{NN}} / k_{B}$ $=1345 \mathrm{~K}$ and $J_{\mathrm{CuAl}}^{\mathrm{NNN}} / k_{B}=825 \mathrm{~K}$, and Ref. 23 gives $J_{\mathrm{CuAl}}^{\mathrm{NN}} / k_{B}$ $=1605 \mathrm{~K}$ and $J_{\mathrm{CuAl}}^{\mathrm{NNN}} / k_{B}=856 \mathrm{~K}$. This agreement shows that these exchange energies are not only parameters obtained for a given model, but they also have a physical meaning. Nevertheless, it has to be pointed out that a complete realistic model should take into account that exchange energies themselves can depend on composition, and this fact is not considered in these simplified models.

From the results of the simulations, we can obtain information about the distribution of the different atomic species in each of the sublattices. This information cannot easily be obtained from the experiments: $\mathrm{x}$-ray diffraction superstructure peaks give information mainly about the occupation of the different sublattices by $\mathrm{Al}$ atoms, but not by $\mathrm{Mn}$, since the scattering factor for $\mathrm{Mn}$ is very similar to that for $\mathrm{Cu}$. $\mathrm{An}$ analysis of the results of the simulations shows that, for alloys with low at. \% $\mathrm{Mn}$ at a temperature just below the $L 2_{1} \rightleftharpoons A 2$ transition, Mn atoms are highly disordered and distributed quite randomly among the different sublattices. Nevertheless, a preference of these atoms to be located mainly on its own sublattice but also on the $\mathrm{Al}$ sublattice is detected. This effect is enhanced as the concentration in-

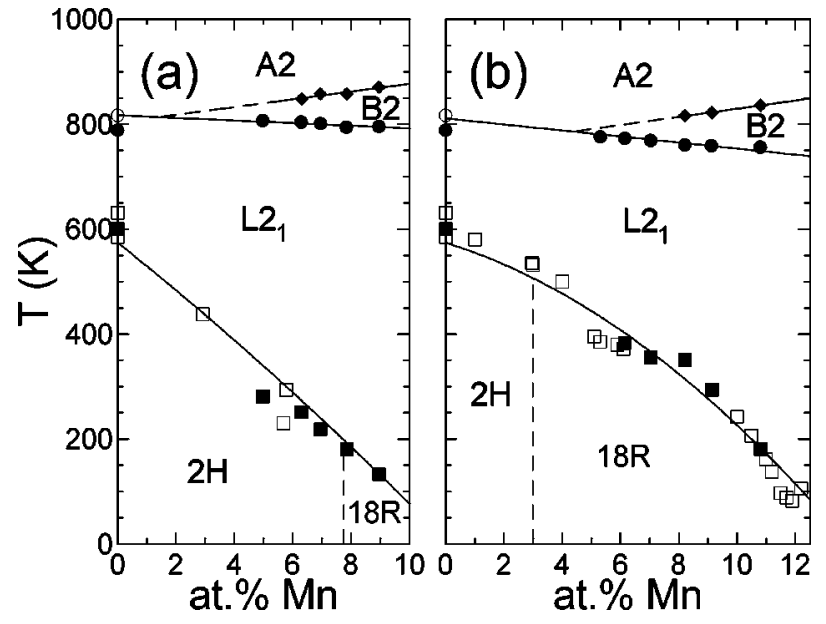

FIG. 7. Metastable phase diagram of $\mathrm{Cu}-\mathrm{Al}-\mathrm{Mn}$, (a) along the $\mathrm{Cu}_{3} \mathrm{Al} \rightarrow \mathrm{Mn}$ line, and (b) along the $\mathrm{Cu}_{3} \mathrm{Al} \rightarrow \mathrm{Cu}_{3} \mathrm{Mn}_{2}$ line. Solid symbols: measurements from present work and a previous study using the same samples (Ref. 15). Open symbols: values taken from the literature. Lines are fits to the experimental data. $L 2_{1}\left(D O_{3}\right) \rightleftharpoons A 2$ and $L 2_{1} \rightleftharpoons B 2$ transition temperatures. $\bullet$ : $B 2 \rightleftharpoons A 2$ transition temperatures. $\mathbf{\square}$ : martensitic transition $\left(M_{s}\right)$ temperatures. Dashed vertical lines indicate the border between $2 H$ and $18 R$ martensites.

creases, and demonstrates the tendency of $\mathrm{Mn}$ atoms to favor $B 2$ ordering, which appears when the Mn concentration is high enough. This fact is obviously due to the particular set of exchange energies between the different atomic species of the system. It is worth noting that we have recently shown ${ }^{29}$ that these results are also relevant to understand the magnetic properties of $\mathrm{Cu}-\mathrm{Al}-\mathrm{Mn}$ alloys, which strongly depend on the distribution of $\mathrm{Mn}$ atoms in the crystal.

\section{PHASE DIAGRAM}

In addition to the equilibrium phase diagram shown in Fig. 1, the metastable phase diagram, i.e., the phase diagram when the bcc phase is retained at low temperature, has been obtained. The temperatures of the martensitic transformation of the alloys studied in this paper have been recently reported in Ref. 15: The complete metastable phase diagram is plotted in Fig. 7. From this figure, it can be noticed that the martensitic transformation temperatures show a strong composition dependence, while the order-disorder transition temperatures appear to be practically independent on composition.

It must be pointed out that the diagrams of Figs. 1 and 7 hold for the same alloys, so that both must be considered simultaneously when determining the properties of $\mathrm{Cu}-\mathrm{Al}-$ Mn. This assertion is especially significant for the orderdisorder transitions, which occur at a range of temperatures where precipitation of equilibrium phases is likely to occur. We showed in Fig. 4 the order-disorder transitions together with the phase borders of the stable phases. It can be observed that the $L 2_{1} \rightleftharpoons B 2$ transition line for samples belonging to the $\mathrm{Cu}_{3} \mathrm{Al} \rightarrow \mathrm{Cu}_{3} \mathrm{Mn}_{2}$ family is very close to the lowest limit of stability of the $\beta$ phase. In fact, these are the samples for which an interaction between the order-disorder transition and the precipitation process has been observed. 


\section{SUMMARY AND CONCLUSIONS}

A calorimetric study of the order-disorder phase transitions in $\mathrm{Cu}-\mathrm{Al}-\mathrm{Mn}$ has been performed. It has been shown that two continuous transitions occur, $L 2_{1} \rightleftharpoons B 2$ and $B 2 \rightleftharpoons A 2$; these two transitions join in a single first order $\mathrm{DO}_{3} \rightleftharpoons A 2$ transition for low Mn contents. The temperatures for all these transitions have been measured. The energy changes at the order-disorder transitions have also been computed.

It has been observed that, contrarily to what occurs in other $\mathrm{Cu}$-based alloys, such as $\mathrm{Cu}-\mathrm{Zn}-\mathrm{Al}$, it is very difficult to retain frozen disorder at room temperature in $\mathrm{Cu}-\mathrm{Al}-\mathrm{Mn}$. This is an important result because martensitic transformation properties are strongly influenced by the degree of disorder retained by the samples. It has also been shown that ordering has a stabilizing effect on the bcc phase of $\mathrm{Cu}$-based alloys, in the sense that it drastically slows down the kinetics of precipitation of equilibrium phases.

It has been found that the main general features (temperatures, heat capacities, and order of the transitions) of the order-disorder transitions appearing in the phase diagram of $\mathrm{Cu}-\mathrm{Al}-\mathrm{Mn}$ can be reproduced by means of Monte Carlo simulations on a simplified Blume-Emery-Griffiths model with three adjustable parameters. From this model, a first order $L 2_{1} \rightleftharpoons A 2$ transition is found at low Mn contents, which splits into two second order transitions, $L 2_{1} \rightleftharpoons B 2$ and $B 2 \rightleftharpoons A 2$, on increasing the Mn concentration, in agreement with the experimental results, though the Mn content at which this splitting occurs for the simulations is slightly higher than that observed experimentally. The model contributes to the understanding of the experimental observations. It gives information concerning the occupations of the different sublattices, which confirms that the increase in Mn concentration favors the appearance of an intermediate $B 2$ phase. From present results and data reported previously for the martensitic transition of the studied alloy families, the metastable phase diagram of bcc $\mathrm{Cu}-\mathrm{Al}-\mathrm{Mn}$ has been determined.

\section{ACKNOWLEDGMENTS}

Samples were kindly provided by M. Morin, INSA, Lyon (France). X-ray diffraction experiments were performed at SCT (Universitat de Barcelona), with the help of X. Alcobé and J. Bassa. This work has received financial support from CICyT (Spain), project no. MAT98-0315 and from CIRIT (Catalonia), project no. SGR00119. E.O. acknowledges financial support from DGICyT (Spain), and C.F. from D.G.R. (Catalonia).

\section{APPENDIX A}

We investigate the characteristics of the order-disorder transitions of a bcc alloy from symmetry arguments. To simplify the situation, we will consider in this appendix a binary alloy $A_{x} B_{1-x}$, since no new phases are observed experimentally from the introduction of a third element.

We divide the bcc lattice into four fcc sublattices $\alpha, \beta, \gamma$, and $\delta,{ }^{30}$ and introduce a pseudospin variable $S_{i}$ at each lattice site $i$ of the crystal, which takes the values +1 or -1 if the site is occupied by an $A$ or a $\mathrm{B}$ atom, respectively. We define the magnetization $m$ of each sublattice $\mu$ as

$$
m_{\mu}=\sum_{i \in \mu} S_{i}
$$

from these magnetizations, the following three order parameters can be defined: ${ }^{31}$

$$
\begin{gathered}
\xi_{1}=m_{\alpha}+m_{\gamma}-m_{\beta}-m_{\delta}, \\
\xi_{2}=m_{\alpha}-m_{\gamma}+m_{\beta}-m_{\delta}, \\
\xi_{3}=-m_{\alpha}+m_{\gamma}+m_{\beta}-m_{\delta} .
\end{gathered}
$$

This is a complete set of order parameters for a binary alloy. To describe all the possible phases of a ternary alloy, additional order parameters would be required, but a set of three order parameters is enough to characterize the ordered phases of interest in this paper. The disordered $A 2$ phase corresponds to $\xi_{1}=\xi_{2}=\xi_{3}=0$, i.e., $m_{\alpha}=m_{\gamma}=m_{\beta}=m_{\delta}$. If $m_{\alpha}=m_{\gamma} \neq m_{\beta}=m_{\delta}$, then $\xi_{1} \neq 0, \xi_{2}=\xi_{3}=0$, which is the $B 2$ phase. The $B 32$ phase, which does not appear for the samples studied in this work, occurs when $m_{\alpha}=m_{\beta} \neq m_{\gamma}$ $=m_{\delta}\left(\xi_{2} \neq 0, \xi_{1}=\xi_{3}=0\right)$ or $m_{\alpha}=m_{\delta} \neq m_{\beta}=m_{\gamma}\left(\xi_{3} \neq 0, \xi_{1}\right.$ $\left.=\xi_{2}=0\right)$. The $L 2_{1}$ phase corresponds to $m_{\alpha}=m_{\gamma} \neq m_{\beta}$ $\neq m_{\delta}$ or $m_{\alpha} \neq m_{\gamma} \neq m_{\beta}=m_{\delta}$, that is, $\xi_{1} \neq 0,\left|\xi_{2}\right|=\left|\xi_{3}\right| \neq 0$. Finally, the $\mathrm{DO}_{3}$ phase is just a particular case of the $L 2_{1}$ phase, in which the magnetizations of three of the sublattices are equal, and different from the magnetization of the fourth sublattice; that means $\left|\xi_{1}\right|=\left|\xi_{2}\right|=\left|\xi_{3}\right| \neq 0$. $^{32}$

Let $h, k$, and $l$ be three integer numbers. Then, the vector $(h, k, l) 2 \pi / a$ (where $a$ is the lattice parameter of the $L 2_{1}$ ordered phase) will belong to the reciprocal lattice (and consequently will give rise to a Bragg peak in the diffraction pattern of the alloy) if certain conditions are satisfied, which will depend on the state of order of the alloy: Thus, the diffraction pattern of a disordered alloy will contain the $h k l$ Bragg peaks with $h, k, l$ even and $h+k+l=4 n, n \in Z$; these are the fundamental reflections of the system. For an alloy with $B 2$ order, the peaks with $h, k, l$ even and $h+k+l$ $=4 n+2$, which are the $B 2$ superstructure reflections, will also appear. In addition, if the alloy is $L 2_{1}$ or $D O_{3}$ ordered, the reflections with $h, k$, and $l$ odd will also be contained in the pattern. The same superstructure peaks will be present for $L 2_{1}$ and $\mathrm{DO}_{3}$ phases, but the relative intensities of the superstructure maxima obtained in each case will be different. Because of this, the $L 2_{1}$ and $D O_{3}$ phases can, in principle, be distinguished from diffraction data.

In order to study which order-disorder phase transitions are possible in our system and the order of these transitions, we will consider a Landau expansion for the free energy. ${ }^{33}$ The terms appearing in this expansion must be invariant under relabeling of the sublattices. It can be seen that this condition forces the free energy to be invariant (i) under sign changes of whichever two of the three order parameters, and (ii) under the exchange between $\xi_{2}$ and $\xi_{3}$. Then, the free energy expansion (up to fourth order) can only contain the following terms:

$$
\begin{aligned}
F= & F_{0}+a_{1} \xi_{1}^{2}+a_{2}\left(\xi_{2}^{2}+\xi_{3}^{2}\right)+b \xi_{1} \xi_{2} \xi_{3}+c_{1} \xi_{1}^{4}+c_{2} \xi_{2}^{2} \xi_{3}^{2} \\
& +c_{3}\left(\xi_{2}^{4}+\xi_{3}^{4}\right)+c_{4} \xi_{1}^{2}\left(\xi_{2}^{2}+\xi_{3}^{2}\right)
\end{aligned}
$$


where $F_{0}, a_{1}, a_{2}, b, c_{1}, c_{2}, c_{3}, c_{4}$ are functions of the temperature $T$, the pressure $P$, and the concentration variable $x .{ }^{34}$ These coefficients determine the phase transitions of the system, since the allowed values of the order parameters are obtained by minimization of $F\left(T, P, x ; \xi_{1}, \xi_{2}, \xi_{3}\right)$.

For $a_{1}$ and $a_{2}$ positive, the only minimum of the free energy corresponds to $\xi_{1}=\xi_{2}=\xi_{3}=0$, the $A 2$ phase. In case $a_{1}$ goes through 0 to negative values, with $a_{2}$ remaining positive, a transition to $B 2$ phase occurs; this transition will be second order if $c_{1}>0$. If, after this transition, $a_{2}$ goes to 0 , a $B 2 \rightarrow L 2_{1}$ transition can take place; this transition can be second order if $d_{3} \equiv 2 c_{3}+c_{2}>0$. The transition point is determined by the equation $a_{2} \pm \frac{1}{2} b \xi_{1}+c_{4} \xi_{1}^{2}=0$. The sign taken by $\xi_{1}$ determines whether $\xi_{2}=\xi_{3}$ or $\xi_{2}=-\xi_{3}$ in the $L 2_{1}$ phase, because the previous formation of the $B 2$ phase has broken the symmetry among the four sublattices.

If, from the $A 2$ phase ( $a_{1}$ and $a_{2}$ positive), it is $a_{2}$ that goes through 0 while $a_{1}$ remains positive, two kinds of transitions are possible, to $L 2_{1}$ or to $B 32$ phases. There will be a second order $A 2 \rightarrow B 32$ transition if $c_{2}>2 c_{3}>0$, whereas a second order $A 2 \rightarrow L 2_{1}$ transition will take place if $d_{2}>d_{3}$ $>0$, where $d_{2}=6 c_{3}-c_{2}$. An $A 2 \rightarrow L 2_{1}$ transition requires $\xi_{1}$ to change from 0 ; this is made possible, despite $a_{1}>0$, because of the term $b \xi_{1} \xi_{2} \xi_{3}$ : when $\xi_{2}$ and $\xi_{3}$ become different from 0 , this term acts as an ordering field on $\xi_{1}$, and displaces from 0 the value of $\xi_{1}$ that minimizes $F$.

Finally, we consider transitions to the $\mathrm{DO}_{3}$ phase. An $A 2 \rightarrow D O_{3}$ transition is a particular case of the $A 2 \rightarrow L 2_{1}$, where the additional condition $\left|\xi_{1}\right|=\left|\xi_{2}\right|$ is imposed. This additional condition restricts the possibility for a second order $\mathrm{A}_{2} \rightarrow \mathrm{DO}_{3}$ transition to occur, at most, at isolated points of the phase diagram. In general, such a transition will be first order, provided that it exists. $\mathrm{A} \mathrm{B} 2 \rightarrow \mathrm{DO}_{3}$ transition can only exist as a first order transition too, since it can only happen through a discontinuous change of $\xi_{2}$ and $\xi_{3}$ : from $\xi_{1} \neq 0, \xi_{2}=\xi_{3}=0$ to $\left|\xi_{1}\right|=\left|\xi_{2}\right|=\left|\xi_{3}\right| \neq 0$.

If, in the phase diagram of an alloy, there is a region with two second order transitions, $A 2 \rightarrow B 2$ and $B 2 \rightarrow L 2_{1}$, it is possible that these two transitions join in a unique phase transition at some point of the phase diagram. This transition may be $A 2 \rightarrow D O_{3}$, which will be first order, or $A 2 \rightarrow L 2_{1}$, which will be second order if $d_{2}>d_{3}$ or first order otherwise. Previous to the joining of the two phase transitions, it is also possible that the $B 2 \rightarrow L 2_{1}$ transition becomes first order. These results are in agreement with the experimental results and computer simulations published by several authors ${ }^{31,35}$ on $\mathrm{Fe}-\mathrm{Al}$ and $\mathrm{Fe}-\mathrm{Si}$ systems. It has to be noticed, however, that all those works found the $A 2 \rightarrow L 2_{1}$ transitions to be first order. ${ }^{36}$ First order $\mathrm{A} 2 \rightarrow \mathrm{DO}_{3}$ transitions have also been found in the $\mathrm{Cu}_{3} \mathrm{Al}(\mathrm{Be})$ system, ${ }^{21}$ and, up to this date, we do not know any experimental or simulation result finding an $A 2 \rightarrow L 2{ }_{1}$ transition of second order in any alloy.

\section{APPENDIX B}

Let us consider a nearest neighbor (NN) and next-nearest neighbor (NNN) pair interaction model between the three kinds of atoms, $A, B$, and $C$, of a ternary alloy $A_{1-x-y} B_{x} C_{y}$. The configurational energy of this alloy will be given by the Hamiltonian

$$
H=\sum_{\langle\alpha \beta\rangle}^{\mathrm{NN}} N_{\alpha \beta}^{\mathrm{NN}} \epsilon_{\alpha \beta}^{\mathrm{NN}}+\sum_{\langle\alpha \beta\rangle}^{\mathrm{NNN}} N_{\alpha \beta}^{\mathrm{NNN}} \epsilon_{\alpha \beta}^{\mathrm{NNN}}
$$

where $\alpha, \beta=A, B$, or $C ; N_{\alpha \beta}^{\mathrm{NN}}$ and $N_{\alpha \beta}^{\mathrm{NNN}}$ are the number of $\alpha-\beta$ pairs in the crystal that are NN or NNN, respectively; and $\varepsilon_{\alpha \beta}^{\mathrm{NN}}$ and $\varepsilon_{\alpha \beta}^{\mathrm{NNN}}$ are the effective pair interaction energies between an $\alpha$ and a $\beta$ atom. Usually, exchange energies for an $\alpha-\beta$ pair $(\alpha, \beta=A, B, C, \alpha \neq \beta)$ are defined as

$$
\begin{gathered}
J_{\alpha \beta}^{\mathrm{NN}}=\varepsilon_{\alpha \alpha}^{\mathrm{NN}}+\varepsilon_{\beta \beta}^{\mathrm{NN}}-2 \varepsilon_{\alpha \beta}^{\mathrm{NN}} \\
J_{\alpha \beta}^{\mathrm{NNN}}=\varepsilon_{\alpha \alpha}^{\mathrm{NNN}}+\varepsilon_{\beta \beta}^{\mathrm{NNN}}-2 \varepsilon_{\alpha \beta}^{\mathrm{NNN}}
\end{gathered}
$$

It can be seen that, introducing the pseudospin variable $S_{i}$, which takes the value $+1,-1$ or 0 if the lattice site $i$ is occupied by an $A$, a $B$, or a $C$ atom, respectively, Hamiltonian (B1) is equivalent to the Blume-Emery-Griffiths ${ }^{26}$ Hamiltonian of Eq. (2), where $J_{1}, J_{2}, K_{1}, K_{2}, L_{1}$, and $L_{2}$, are parameters related to the exchange energies:

$$
\begin{gathered}
J_{1}=\frac{1}{4} J_{A B}^{\mathrm{NN}}, \\
J_{2}=\frac{1}{4} J_{A B}^{\mathrm{NN}}, \\
K_{1}=\frac{1}{4}\left(2 J_{A C}^{\mathrm{NN}}+2 J_{B C}^{\mathrm{NN}}-J_{A B}^{\mathrm{NN}}\right), \\
K_{2}=\frac{1}{4}\left(2 J_{A C}^{\mathrm{NNN}}+2 J_{B C}^{\mathrm{NNN}}-J_{A B}^{\mathrm{NNN}}\right), \\
L_{1}=\frac{1}{4}\left(J_{A C}^{\mathrm{NN}}-J_{B C}^{\mathrm{NN}}\right), \\
L_{2}=\frac{1}{4}\left(J_{A C}^{\mathrm{NNN}}-J_{B C}^{\mathrm{NNN}}\right) .
\end{gathered}
$$

In our simulations, we identified the $A$ species with $\mathrm{Cu}, B$, with $\mathrm{Al}$, and $C$ with $\mathrm{Mn}$. We took the minimum number of parameters $\left(J_{1}, J_{2}\right.$, and $\left.L_{2}\right)$ necessary to obtain the ordered phases observed experimentally in the system studied, and we assumed the remaining parameters to be 0 . This is expected to be a good approximation if the terms containing $J_{1}, J_{2}$, and $L_{2}$ have a higher absolute value than the remaining terms. In this case, considering the complete model with $K_{1}, K_{2}$, and $L_{1}$ terms different from 0 would only introduce corrections to the temperatures and energy changes obtained for the transitions, but would not modify the general features of the phase diagram obtained with this simplified model.

Monte Carlo simulations were carried out in bec lattices with up to $2 \times 64^{3}\left(\sim 5 \times 10^{5}\right)$ atoms and periodic boundary conditions. The standard Kawasaki dynamics was introduced using the Metropolis algorithm. The transition temperatures, at each composition, were found through "heating" processes. These processes began at a temperature below the transition point and with the atoms forming an $L 2_{1}$ super- 
structure $\left(\mathrm{DO}_{3}\right.$ in the $\mathrm{Cu}_{3} \mathrm{Al}$ case). Once the equilibrium was reached, we extracted from the simulation the configurational energy and the magnetizations defined in Eq. (A1). Then the temperature was increased and the simulation con- tinued until the equilibrium was reached again. This last step was repeated until the transition line (lines) was (were) crossed. Hysteresis in the first order phase transitions was studied performing equivalent "cooling'" processes.
*Present address: Institut de Ciència de Materials de Barcelona, CSIC Campus Universitat Autònoma de Barcelona, E-08193 Bellaterra, Catalonia, Spain.

${ }^{1}$ T. B. Massalski and U. Mizutani, Prog. Mater. Sci. 22, 151 (1978).

${ }^{2}$ M. Ahlers, Prog. Mater. Sci. 30, 135 (1986); R. Romero and J. L. Pelegrina, Phys. Rev. B 50, 9046 (1994).

${ }^{3}$ L. Delaey, in Materials Science and Technology, edited by P. Haasen (VCH, Weinheim, 1991), Vol. 5, p. 339.

${ }^{4}$ H. Kato, J. Dutkiewicz, and S. Miura, Acta Metall. Mater. 42, 1359 (1994).

${ }^{5}$ D. R. F. West and D. Lloyd Thomas, J. Inst. Met. 85, 97 (1956).

${ }^{6}$ F. Heusler, W. Starck, and E. Hapt, Verh. Dtsch. Phys. Ges. 5, 220 (1903)

${ }^{7}$ G. B. Johnston and E. O. Hall, J. Phys. Chem. Solids 29, 201 (1968).

${ }^{8}$ K. Tajima et al., J. Phys. Soc. Jpn. 43, 483 (1977).

${ }^{9}$ J. J. Conioux, J. L. Macqueron, M. Robin, and J. M. Scarabello, Scr. Metall. 22, 821 (1988); J. M. Scarabello, Ph.D. thesis, Université Claude-Bernard, Lyon, 1984.

${ }^{10}$ J. Soltys, Phys. Status Solidi A 63, 401 (1981).

${ }^{11}$ M. Bouchard and G. Thomas, Acta Metall. 23, 1485 (1975).

${ }^{12}$ T. V. Yefimova, V. V. Kokorin, V. V. Polotniuk, and A. D. Shevchenko, Phys. Met. Metallogr. 64, 189 (1987); L. Yiping, A. Murthy, G. C. Hadjipanayis, and H. Wan, Phys. Rev. B 54, 3033 (1996).

${ }^{13}$ K. Matsushita, T. Okamoto, and T. Okamoto, J. Mater. Sci. 20, 689 (1985); D. Roqueta, F. C. Lovey, and M. Sade, Scr. Mater. 34, 1747 (1996).

${ }^{14}$ N. Nakanishi et al., in Proceedings of ICOMAT'92, edited by C. M. Wayman and J. Perkins (Monterey Institute of Advanced Studies, Carmel, CA, 1993), p. 581.

${ }^{15}$ E. Obradó, Ll. Mañosa, and A. Planes, Phys. Rev. B 56, 20 (1997), and references reported therein.

${ }^{16}$ The eutectoid point is the point where the $\beta$ phase is stable to lowest temperatures.

${ }^{17}$ Ll. Mañosa, M. Jurado, A. Gonzàlez-Comas, E. Obradó, A. Planes, J. Zaretsky, C. Stassis, R. Romero, A. Somoza, and M. Morin, Acta Mater. 46, 1045 (1998).

${ }^{18}$ This volume change arises from two contributions: (i) the thermal expansion and (ii) the disordering of the alloy.
${ }^{19}$ Some values corresponding on cooling are lacking in Table III, because a good baseline above or below the phase transition could not be obtained.

${ }^{20}$ G. Roulin and P. Duval, Scr. Mater. 37, 45 (1997); B. Predel, in Landolt-Börnstein New Series, Vol. IV/5a, edited by O. Madelung (Springer-Verlag, Berlin, 1991), p. 160; J. L. Murray, in Binary Alloy Phase Diagrams, edited by T. B. Massalski (American Society for Metals, Ohio, 1986), Vol. 1, p. 103; P. R. Swann and H. Warlimont, Acta Metall. 11, 511 (1963).

${ }^{21}$ M. Jurado, T. Castan, Ll. Mañosa, A. Planes, J. Bassas, X. Alcobé, and M. Morin, Philos. Mag. A 75, 1237 (1997).

${ }^{22}$ R. Rapacioli and M. Ahlers, Scr. Metall. 11, 1147 (1977).

${ }^{23}$ M. Prado, F. Sade, and F. Lovey, Scr. Metall. Mater. 28, 545 (1993).

${ }^{24}$ Precipitation after the disordering is also observed in $\mathrm{Cu}_{3} \mathrm{Al}(\mathrm{Be})$ : see Fig. 1(a) in Ref. 17.

${ }^{25}$ K. Binder, Rep. Prog. Phys. 60, 487 (1997).

${ }^{26}$ M. Blume, V. J. Emery, and R. B. Griffiths, Phys. Rev. A 4, 1071 (1971).

${ }^{27}$ J. Ni and B. Gu, Phys. Rev. Lett. 79, 3922 (1997).

${ }^{28}$ It has to be noticed, however, that simulations result in a $\mathrm{DO}_{3}$ phase only for stoichiometric $\mathrm{Cu}_{3} \mathrm{Al}$, giving transitions to an $L 2$, phase in all the remaining cases.

${ }^{29}$ E. Obradó, A. Planes, and B. Martínez (unpublished).

${ }^{30}$ Figures showing these sublattices can be found, e.g., in Refs. 21,31 .

${ }^{31}$ B. Dünweg and K. Binder, Phys. Rev. B 36, 6935 (1987); W. Helbing, B. Dünweg, K. Binder, and D. P. Landau, Z. Phys. B 80, 401 (1990).

${ }^{32}$ The $L 2_{1}$ and $D O_{3}$ phases are not distinguished in a great number of papers, which use $\mathrm{DO}_{3}$ to denote both structures.

${ }^{33}$ L. D. Landau, E. M. Lifshitz, and L. P. Pitaevskii, Statistical Physics (Pergamon, Oxford, 1980), Pt. 1, p. 446.

${ }^{34}$ The term with $c_{4}$ was not included in $F$ in Ref. 31. Such a term can be present in the free energy expansion, though it will be seen that it does not affect the stability among the different ordered phases.

${ }^{35}$ D. A. Contreras-Solorio, F. Mejía-Lira, J. L. Morán-López, and J. M. Sánchez, Phys. Rev. B 38, 16 (1988); J. M. Bell, Physica A 142, 22 (1987).

${ }^{36}$ Those works did not distinguish between $L 2_{1}$ and $D O_{3}$ phases. 